\title{
A Novel Method for Music Recommendation using Social Media Tags
}

\author{
Gunjan Advani \\ Computer Engineering Dept. \\ Sardar Vallabhbhai Patel \\ Institute of technology, \\ Vasad, Gujarat, India
}

\author{
Neha Soni \\ Assistant Professor \\ Computer Engineering Dept. \\ Sardar Vallabhbhai Patel \\ Institute of technology, \\ Vasad, Gujarat, India
}

\begin{abstract}
Tremendous growth of online music data has given new opportunities for building more effective music recommender systems. These systems help users to find and categorize songs according to their likings. The main goal of Recommender Systems (RS) is to predict ratings of items that the users would be interested in. With the rapid development of the Collaborative Tagging approach, tags could be fascinating and helpful information to enrich RS systems. Attributes are the "global" depictions of items while tags are "local" depictions of items provided by the users

Explicit feedback and implicit feedback demonstrates distinct properties of users' preferences with both advantages and disadvantages. Combination of these in a user preference model not only exhibits a number of disputes but can also overwhelm the problems related with each other. Hence, to take advantage of tagging data and see whether better recommendations are generated or not, a novel method for music recommendation is proposed that combines implicit feedback and explicit feedback of the users. Also, both explicit types of feedbacks are normalized before transformation into ratings in order to provide the desired ratings in case of skewed play counts data.
\end{abstract}

\section{General Terms}

Music Recommendation System, Collaborative Tagging

\section{Keywords}

Social media tags, Recommendation by Tag-driven Item Similarity

\section{INTRODUCTION}

Music has become most important part in an individual's life for entertainment. A large amount of music content is available to lots of users around the world as the fame of music and Internet technologies has increased. It is becoming increasingly difficult for the users to search for music content, as there are millions of artists and songs in the market. Moreover, huge amounts of music data available have opened new prospects for researchers working on music recommendation to create new feasible services that support music searching and sharing. The requirement for music recommender systems - is important due to the economic potential of online music content [1].

Also, music recommender is to help users filter and discover songs according to their tastes [2]. For now, the growth of recommender systems provides a huge prospect for industry to combine the users who are interested in music. More importantly, it raises challenges for us to better understand and model users' preferences in music [3].

Music recommender systems are decision support tools that reduce the information overload by retrieving only items that are reckoned as relevant for the user, based on the user's profile, i.e., a representation of the user's music preferences [4]. For example, Last.fm [5] - a popular Internet radio and recommender system - allows a user to choose songs or artists as favorites. It also tracks the user's listening behavior, and based on this information can identify and recommend music content that is more likely to be interesting to the user.

Presently, based on users' listening behavior and historical ratings, collaborative filtering algorithm has been found to perform well [6]. Combined with the use of content-based model, the user can get a list of similar songs by low level acoustic features such as rhythm, pitch or high-level features like genre, instrument etc. [7].

However, most of the available music recommender systems recommend music without taking into consideration the user's context, e.g., mood, or current location and activity [8]. In fact, a study on users' musical information needs [9] showed that people often seek music for a certain occasion, event, or an emotional state. In response to these observations, in recent years a new research topic of contextual (or situational) music recommendation has emerged.

Collaborative tagging systems, also known as folksonomies are web-based systems that allow users to label the resources with arbitrary words, so-called tags [10]. These systems are becoming more popular among users these days. For example popular web services such as Flickr, del.icio.us, Last.fm, Gmail, etc, provide prospect for users to tag or label an item of interest. On the whole, tagging is becoming the new trend making easy for the people to easily add metadata to content and is associated to the Web 2.0. Thus, these metadata can be used to enhance search approaches or provide personalized recommendations that satisfy the users' preferences. An item always has the same properties among all users. Alternatively, tags are provided by several users. Thus, tags are associated to the items as well as the users. Even though properties and tags are both metadata and could function as additional background knowledge to enhance RS algorithms, they should be dealt in a different way.

Thus, music recommendation systems can be classified into five categories such as illustrated in the Figure 1[11]. 


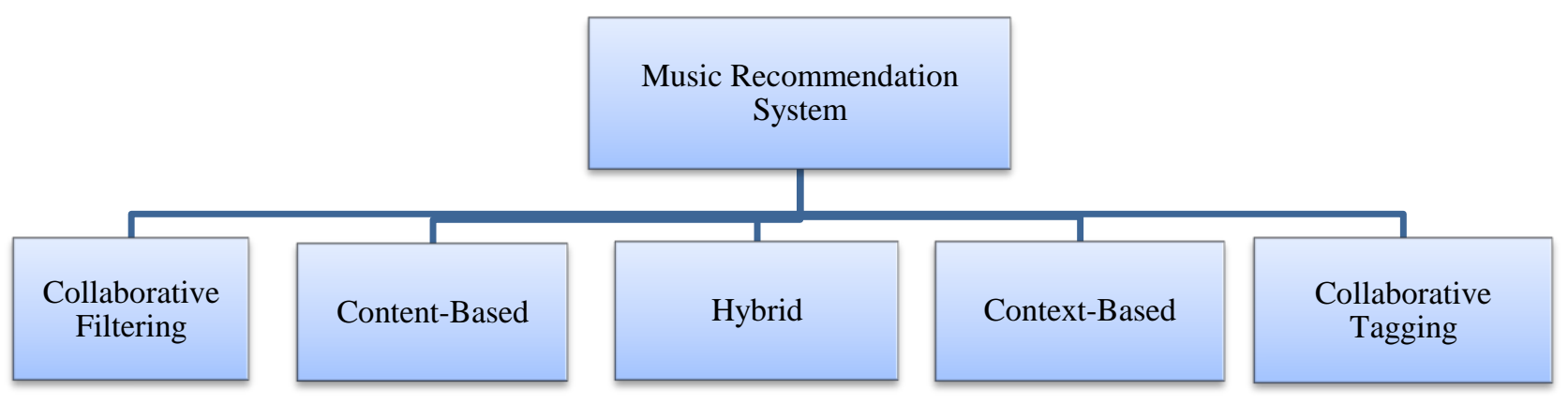

Figure 1 Music Recommendation System Methods

A review of previous work is given in Section 2. In Section 3, we describe the proposed method for predicting the user's preferences by mining social tags. Empirical evaluations of our proposed method on real data set are described in Section 4. Finally, conclusions and future work are stated in Section 5.

\section{RELATED WORK}

Ja-Hwung $\mathrm{Su}$ et al. [12] have proposed a different music recommendation approach that makes use of social media tags instead of ratings to calculate the similarity between music tracks. They used tag-based similarity for finding the user preferences hidden in. It was called Recommendation by Tagdriven Item Similarity (RTIS). This approach also alleviates the problem of rating diversity which was associated with Collaborative Filtering (CF) Recommender Systems.

It takes play counts as implicit ratings and item tags as semantic preferences. The method used for this system is Content-based CF. The experimental results showed that the RTIS method using social tags performs better than the existing ones using only ratings in terms of predicting the user's preferences to music.

Tso Sutter et al. [13] proposed that tags should be incorporated to standard Collaborative filtering (CF) algorithms such as User-and Item based CF. Further, they found an approach that dealt with the 3-dimensional correlation between the users, items and tags. It first applied tag extension mechanism and then a fusion method which had been adapted from a predicting rating problem to predicting item problem.

Further, they studied the effect of incorporating tags information to different $\mathrm{CF}$ algorithms. Leave-one-out protocol was used to evaluate the obtained recommendations. Experimental evaluations were carried on three $\mathrm{CF}$ algorithms with last.fm data set, which demonstrated that incorporating tags to proposed method provided promising and significant results.

Symeonidis et al. [10] attempted to capture the music perception of individual taggers by modeling music items, tags, and users as triplets. The triplets were stored as a 3order tensor. Since such representation of tagging data is sparse, dimensionality reduction was applied. The authors used high order Singular Value Decomposition (SVD). They modeled the social tagging system as a 3-dimensional dataset; the approach relied on predicting the likelihood of a user labeling a certain item with a given tag.

For the evaluation, the authors used the Last.fm dataset, with only positive tags (i.e., eliminating tags that describe negative attitude of taggers towards the items). The available dataset at the time of evaluation consisted of more than 12,000 user-tagartist triples. The authors used a subset of this dataset by keeping those users, tags, and items that appear in at least 5 triples. Since only positive tags were used, the goal of the system is to predict any relevant tag-item pairs for a user. Intuitively, if a user is likely to assign a positive tag to a music item, this item is a relevant recommendation. The evaluation results showed the precision of around $50 \%$ at a similar recall level.

Qi Qi et al. [14] proposed to describe users by the inferred user-to-tag ratings so as to improve user-based $\mathrm{CF}$. Experiments were performed on the MovieLens data set to evaluate the performance of this approach. The results showed that it outperforms traditional user-based collaborative filtering.

Gawesh Jawaheer et al. [15] showed an overview of the differentiating characteristics of explicit and implicit feedback using datasets mined from Last.fm, an online music station is provided. The dataset consisted of explicit positive feedback (through loved tracks) and implicit positive feedback (play count of tracks). Three techniques namely, absolute, normalize and log were presented for extracting user preferences from both types of feedback. For comparing and contrasting the performances of these techniques, experiments were carried out using Taste recommender system engine. Collaborative filtering (CF) algorithm was used to generate the recommendations. Setup was carried out for user-based $\mathrm{CF}$, using a nearest neighborhood value of 3 and Pearson Correlation as the measure of similarity. The outcome of each experiment was measured in terms of the Root Mean Squared Error (RMSE) between the given user preferences and the predicted user preferences. For each experiment, five runs were performed and the results were averaged.

Experiments showed that although they have different characteristics, the two datasets produced similar performances. The aim of the authors in studying explicit and implicit feedback was to better understand their characteristics in order to combine them effectively in a RS.

In [16], the authors looked at histories of tracks loved and tracks played for a sample of users. The analysis showed that the rate of providing explicit feedback by a user decreases over time and that overall providing explicit feedback has a negative effect on the user's behavior.

\section{BASIC IDEA}

Up till now most of the systems have used, implicit feedback as implicit ratings. In this proposed work, implicit feedback (i.e. play counts) and explicit feedback (i.e. love counts) of the user are used to form combination of implicit and explicit ratings. The main focus of this work is to see whether the combination of both feedbacks in the existing system, would result in more effective recommendation system or not. 
Also, both explicit and implicit feedbacks would be normalized before transformation into ratings. This is done in order to provide the desired ratings in case of skewed play counts data. The normalization technique that will be used is Min-Max Normalization. The proposed framework is illustrated in the figure.

As shown in Figure 2, the framework of our proposed recommender system is divided into two stages, namely offline preprocessing and online prediction stages as given in [8]: 1.) Offline preprocessing stage: the purpose of this stage is to accelerate the prediction and to meet the requirement of

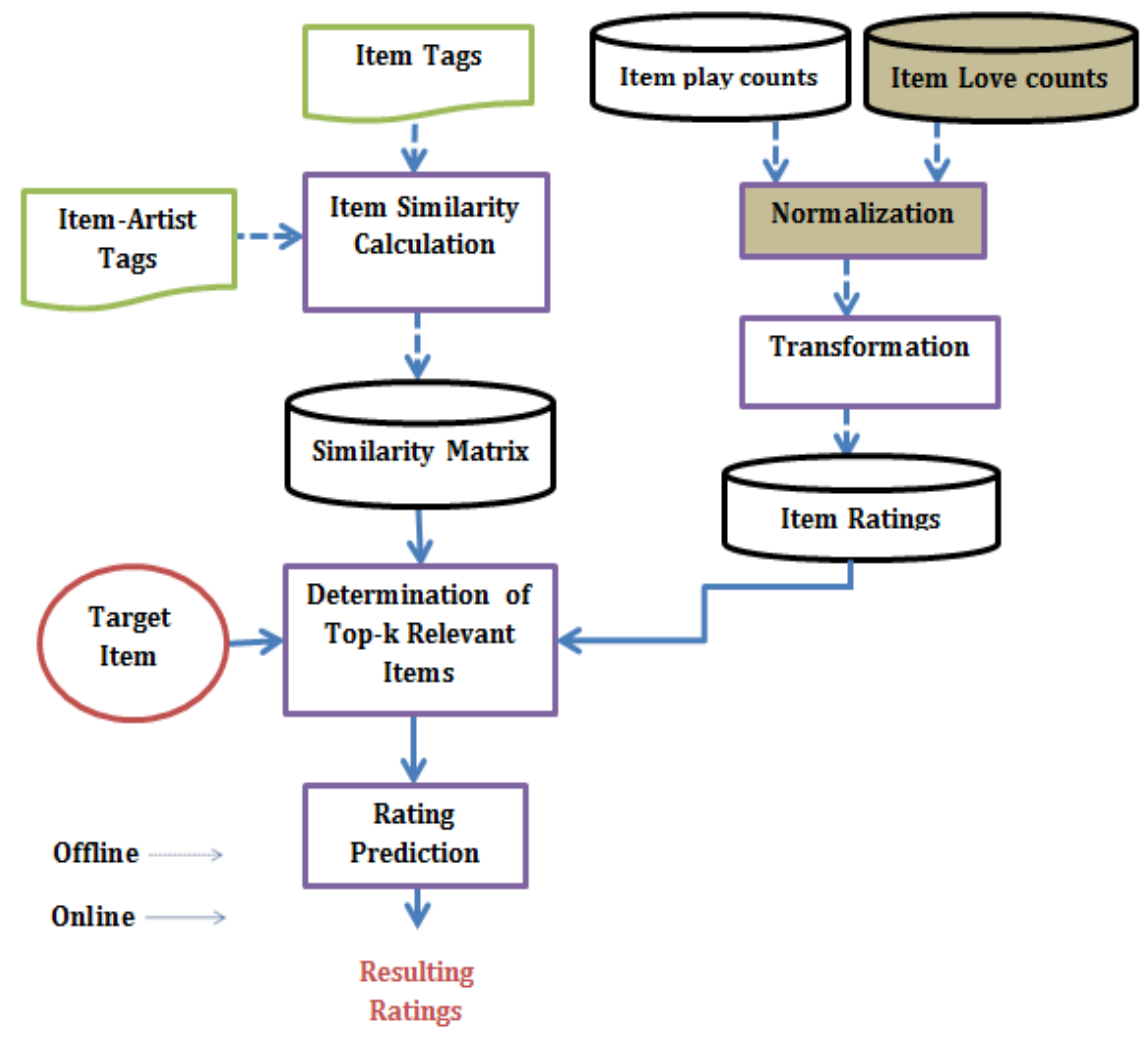

representing the user's preferences by ratings. Hence, the item-ratings and item-similarity-matrix are generated by rating transformation and item similarity calculation, respectively, and 2.) Online prediction stage: this stage is triggered by an active user's visit. For an active user, un-rated items are viewed as target items and then the related item ratings are predicted by referring to other relevant items.

Figure 2 Workflow of proposed system

\section{ALGORITHM}

Step 1: Rating transformation

- Add play counts and love counts

- Normalize the result using Min-Max normalization $v^{\prime}=\frac{v-\min _{A}}{\max _{A}-\min _{A}}\left(n e w_{-} \max _{A}-n e w_{-} \min _{A}\right)+n e w_{-} \min _{A}$

Where minA and maxA are the minimum and maximum values of an attribute, $A$.

It maps a value, $v$, of $A$ to $v$ ' in the range [new minA;new $\max \mathrm{A}]$.

- The play counts are divided into two ranges by a threshold $\mathrm{T}$, which is defined as:

$$
\mathrm{T}=\mu-\tau * \sigma
$$

where $\mu$ indicates mean of play counts, $\sigma$ indicates standard deviation of play counts for a user and $\tau$ is a weight.

- The range lower than $\mathrm{T}$ is further divided into two equivalent sub-ranges with respect to the range number set $\{1,2\}$, while that higher than $\mathrm{T}$ is divided into three equivalent sub-ranges with respect to the range number set $\{3,4,5\}$.

- If a play count is in the specific range, it can be transformed into the referred range number.

Step 2: Construction of item similarity matrix

- Calculate the item-tag-driven similarity between $\mathrm{itm}_{\mathrm{a}}$ and $\mathrm{itm}_{\mathrm{b}}$, given $\mathrm{i}$ unique items $\mathrm{IM}=$ $\left\{\right.$ itm $_{1}$, itm $_{2}, \ldots .$. itm $\left._{i}\right\}, \mathrm{j}$ unique tags $\mathrm{TG}=\left\{\operatorname{tag}_{1}, \operatorname{tag}_{2}, \ldots \ldots, \operatorname{tag}_{\mathrm{j}}\right\}$ in the database and the tag feature vector for itm $_{n} \in \boldsymbol{I M}$ as ivn=\{ $\left.f_{1}^{n}, f_{2}^{n}, \ldots, f_{j}^{n}\right\}$ where $f_{j}^{n}$ is the frequency of $\operatorname{tag}_{\mathrm{j}}$ for $\mathrm{itm}_{\mathrm{n}}$, from given formula

$\operatorname{ITsim}_{\mathrm{a}, \mathrm{b}}=\frac{\sum_{\mathrm{o}<q \leq j} f_{q}^{a} * f_{q}^{b}}{\sqrt{\sum_{\mathrm{o}<q \leq j}\left(f_{q}^{a}\right)^{2} *} \sqrt{\sum_{\mathrm{o}<q \leq j}\left(f_{q}^{b}\right)^{2}}}$

- For example, let there be 10 unique items, 5 unique 
tags and 5 unique artists i.e. $\mathrm{i}=10, \mathrm{j}=5$. Therefore, tag feature vector for itm 1 and itm 2 are

$$
\begin{aligned}
1 & =\left\{f_{1}^{1}, f_{2}^{1}, f_{3}^{1}, f_{4}^{1}, f_{5}^{1}\right\} \\
& =\{5,10,7,0,1\} \\
2 & =\{2,4,6,8,1\}
\end{aligned}
$$

- Thus, item similarity between itm1 and itm2 is given as

$$
\mathrm{ITsim}_{1,2}=\frac{(5 * 2)+(10 * 4)+(7 * 6)+(0 * 8)+(1 * 1)}{\sqrt{5^{2}+10^{2}+7^{2}+0^{2}+1^{2}} \sqrt{2^{2}+4^{2}+6^{2}+8^{2}+1^{2}}}
$$$$
=77.33
$$

- Calculate the artist-tag driven similarity between $\operatorname{art}_{\mathrm{a}}$ and $\operatorname{art}_{\mathrm{b}}$, given $\mathrm{m}$ unique artists $\mathrm{AT}=\left\{\operatorname{art}_{1}, \operatorname{art}_{2}, \ldots \ldots \ldots, \operatorname{art}_{\mathrm{m}}\right\}$ in the database and the tag feature vector for $\operatorname{art}_{\mathrm{m}}$ is defined as $\mathrm{av}_{\mathrm{m}}=$ $\left\{d_{1}^{m}, d_{2}^{m}, \ldots, d_{j}^{m}\right\}$, from given formula

$$
\operatorname{ATsim}_{a, b}=\frac{\sum_{0<q<j} d_{q}^{a} * d_{q}^{b}}{\sqrt{\sum_{0<q<j}\left(d_{q}^{a}\right)^{2}} * \sqrt{\sum_{0<q<j}\left(d_{q}^{b}\right)^{2}}}
$$

where $\operatorname{art}_{\mathrm{a}}=\left\{\operatorname{itm}_{\mathrm{x}} \mid \operatorname{itm}_{\mathrm{x}} \in \mathrm{IM}\right\}$ and

$\operatorname{art}_{\mathrm{b}}=\left\{\operatorname{itm}_{\mathrm{y}} \mid \mathrm{itm}_{\mathrm{y}} \in \mathrm{IM}\right\}$

$$
d_{q=}^{a} \frac{\sum_{\text {itm }_{x} \in \text { art }_{a}} f_{q}^{i t m_{x}}}{\mid \text { art }_{a} \mid}
$$

And

$$
d_{q=}^{b} \frac{\sum_{\text {itm }_{y} \in \operatorname{art}_{b}} f_{q}^{i t m_{y}}}{\left|\operatorname{art}_{b}\right|}
$$

- Now, let $\operatorname{art}_{1}=\left\{\operatorname{itm}_{1}, \operatorname{itm}_{2}\right\}$ and $\operatorname{art}_{2}=\left\{\operatorname{itm}_{2}, \mathrm{itm}_{3}\right\}$. So,

$$
d_{1}^{1}=\frac{f_{1}^{i t m_{1}}+f_{1}^{i t m_{2}}}{\left|a r t_{1}\right|}=3.5
$$

$\therefore$ av1 $=\{3.5,7,6.5,4,1\}$

$$
d_{1}^{2}=\frac{f_{1}^{i t m_{2}}+f_{1}^{i t m_{3}}}{\left|a r t_{2}\right|}=1
$$

$$
\mathrm{av}_{2}=\{1,2.5,4.5,5,3.5\}
$$

- Thus, artist similarity between $\operatorname{art}_{1}$ and $\operatorname{art}_{2}$ is given as

$$
\operatorname{ATsim}_{1,2}=\frac{\sum_{0<q<5} d_{q}^{1} * d_{q}^{2}}{\sqrt{\sum_{0<q<5}\left(d_{q}^{1}\right)^{2} *} \sqrt{\sum_{0<q<5}\left(d_{q}^{2}\right)^{2}}}
$$

$=0.834$

- Given an item-tag-driven similarity $\operatorname{ITsim}_{\mathrm{a}, \mathrm{b}}$ and an artist-tag-driven similarity $\mathrm{ATsim}_{\mathrm{a}, \mathrm{b}}$, the fusion similarity between $\operatorname{itm}_{\mathrm{a}}$ and $\mathrm{itm}_{\mathrm{b}}$ can be calculated as

FUsim $_{a, b}=$ ITsim $_{a, b} * A T \operatorname{sim}_{a, b}$

Step 3: Rating prediction

- The goal of this step is to infer the unknown ratings for the active user.

- It starts with an active user's $\mathrm{u}_{\mathrm{z}}$, visit and then the unknown ratings for the active user are predicted one by one.

- First, the prediction determines $\mathrm{k}$ most-relevant items to the target item itm $_{\mathrm{i}}$ by the calculated item similarities which are given as $\mathrm{U}_{\mathrm{z}}=\cup_{i t m_{p}}$, where $\operatorname{itm}^{p} \in \mathrm{IM}$ e.g.: $\mathrm{U}_{1}=\left\{\mathrm{itm}_{1}, \mathrm{itm}_{3}, \mathrm{itm}_{7}\right\}$

- Next, the rating $\mathrm{v}$ is calculated for an unknown target item, itm $_{\mathrm{i}}$ for $\mathrm{u}_{\mathrm{z}}$ as:

$$
v_{i}^{z}=\frac{\sum_{i t m_{p} \in \mathrm{Uz}} \operatorname{sim}_{i, p} * v_{p}^{z}}{\sum_{i t m_{p} \in \mathrm{Uz}} \operatorname{sim}_{i, p}}
$$

where

$\operatorname{sim}_{\mathrm{i}, \mathrm{p}}=\operatorname{ITsim}_{\mathrm{i}, \mathrm{p}}$, if item tag driven similarity is used(IDP) $\operatorname{sim}_{\mathrm{i}, \mathrm{p}}=\mathrm{ATsim}_{\mathrm{i}, \mathrm{p}}$,if artist tag driven similarity is used(ADP)

$\operatorname{sim}_{\mathrm{i}, \mathrm{p}}=\mathrm{FUSim}_{\mathrm{i}, \mathrm{p}}$, if fusion similarity is used(RTIS)

- Therefore, the rating $\mathrm{v}$ is calculated for itm5 for $\mathrm{u} 1$ as follows:

$\therefore v_{5}^{1}=\frac{(66 * 1)+(50 * 3)+(42 * 4)}{(66)+(50)+(42)} \approx 2$

\section{EMPIRICAL STUDY}

The experimental data was gathered from Last.fm. In fact, Last.fm is a popular social music website which provides the users with online listening and tagging.

\subsection{Ratings before and after normalization}

The Graphs presented here shows the comparison of ratings before and after normalization transformed from the playcounts using threshold T. Analysis of these graphs shows that the proposed method which uses normalization outperforms the first method of transformation for the skewed data. It shows that normalization produces more smooth transformation. 


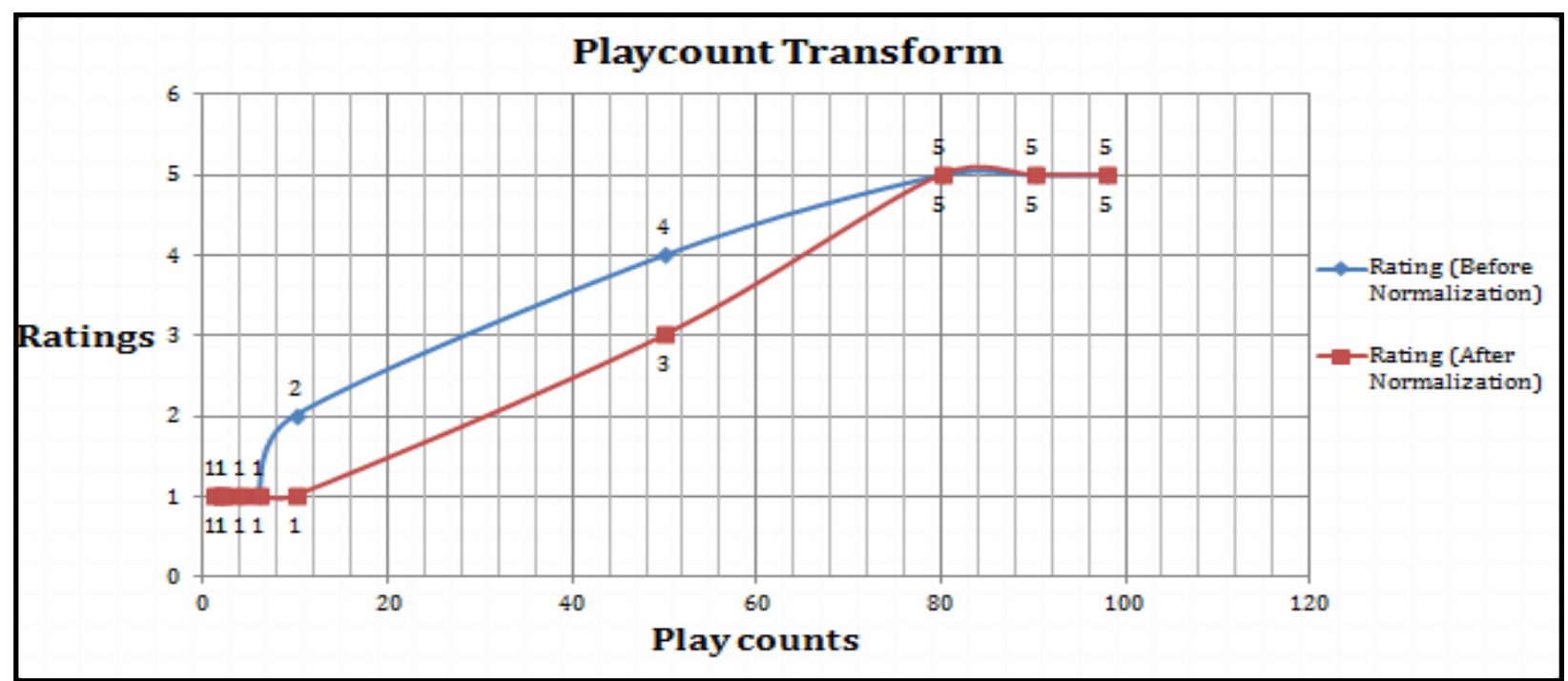

Figure 3 Comparison of ratings before and after normalization of Playcounts

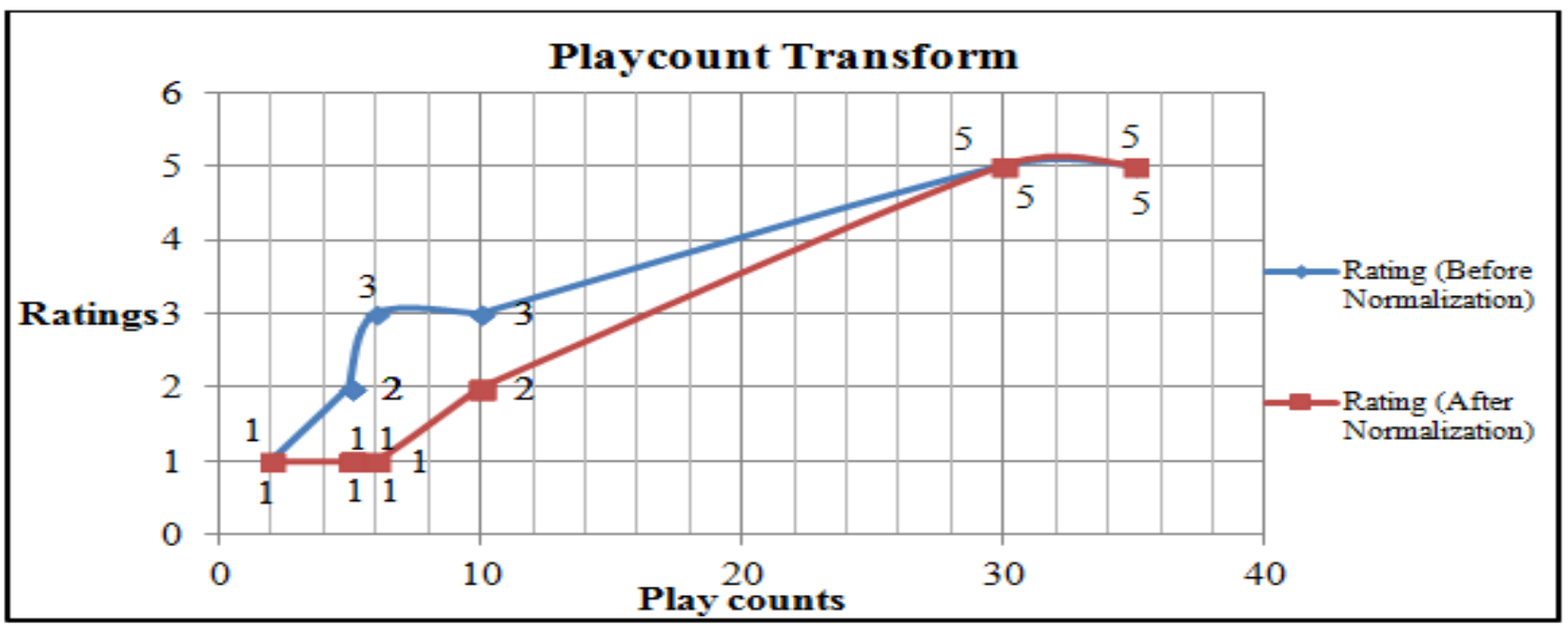

Figure 4 Comparison of ratings before and after normalization of Playcounts

\subsection{Comparison between Different Prediction Models}

To analyze the efficiency of the proposed method, the popular criterion, namely Root Mean Square Error (RMSE), is employed to measure the related experimental performance. It is defined as:

$\operatorname{RMSE}=\sqrt{\frac{\sum_{|t e s t|}(v-\hat{v})^{2}}{|\operatorname{tes} t|}}$

where $\mathrm{v}$ stands for the ground truth, $\hat{v}$ stands for the predicted rating value and test stands for the testing data set. Usually, RMSE shows the error variance. That is, the lower the RMSE, lower is the error, higher the precision and better is the recommendation.

Here in all experiments, the predictions for any active user uz were performed by selecting top $\mathrm{k} \%$ of most-relevant items as Uz stated in step 8 of proposed algorithm. The graphs shown below show the comparison of the methods used in existing system and the methods of our proposed model. From the graphs, there are some observations to be noticed. First, the artist-tag-driven prediction (ADP) model performs better than the item-tag-driven prediction (IDP) model. Second, the fusion model is the best. Third, it could be seen from the second graph that RMSE is lower for all the three methods of the proposed model than the methods of existing method[12]. Therefore, it can be concluded that the proposed model provides better recommendations. 


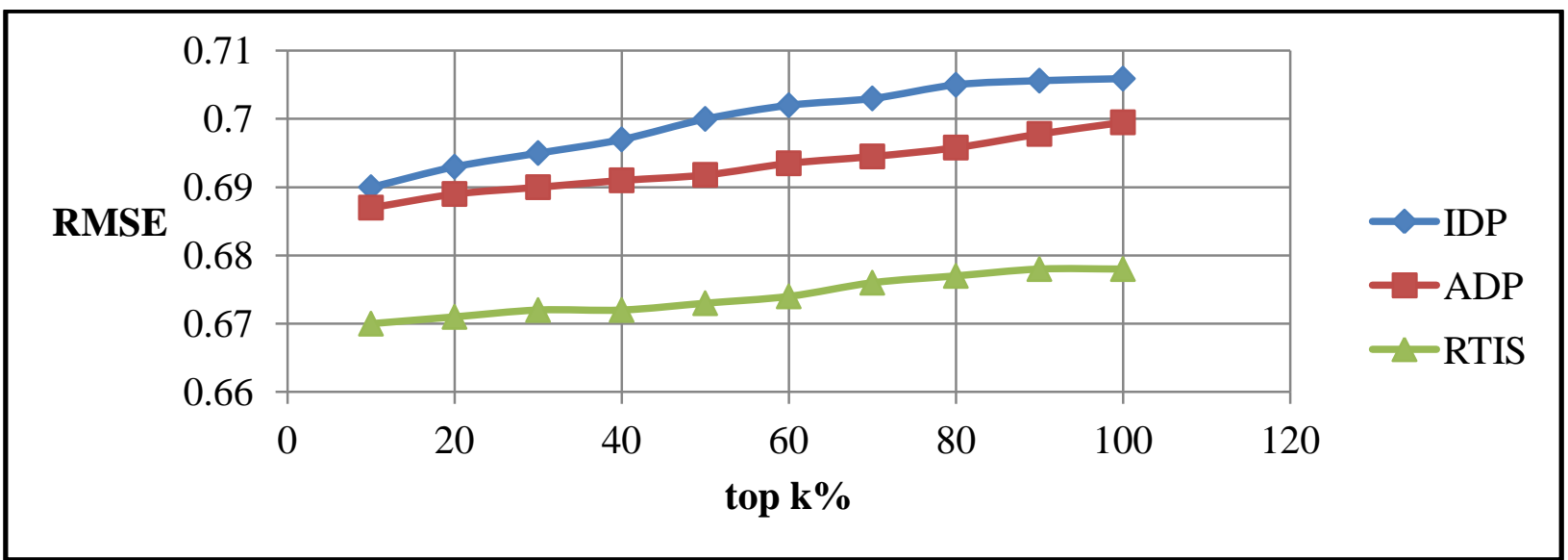

Figure 5 Comparison of different prediction models of existing system

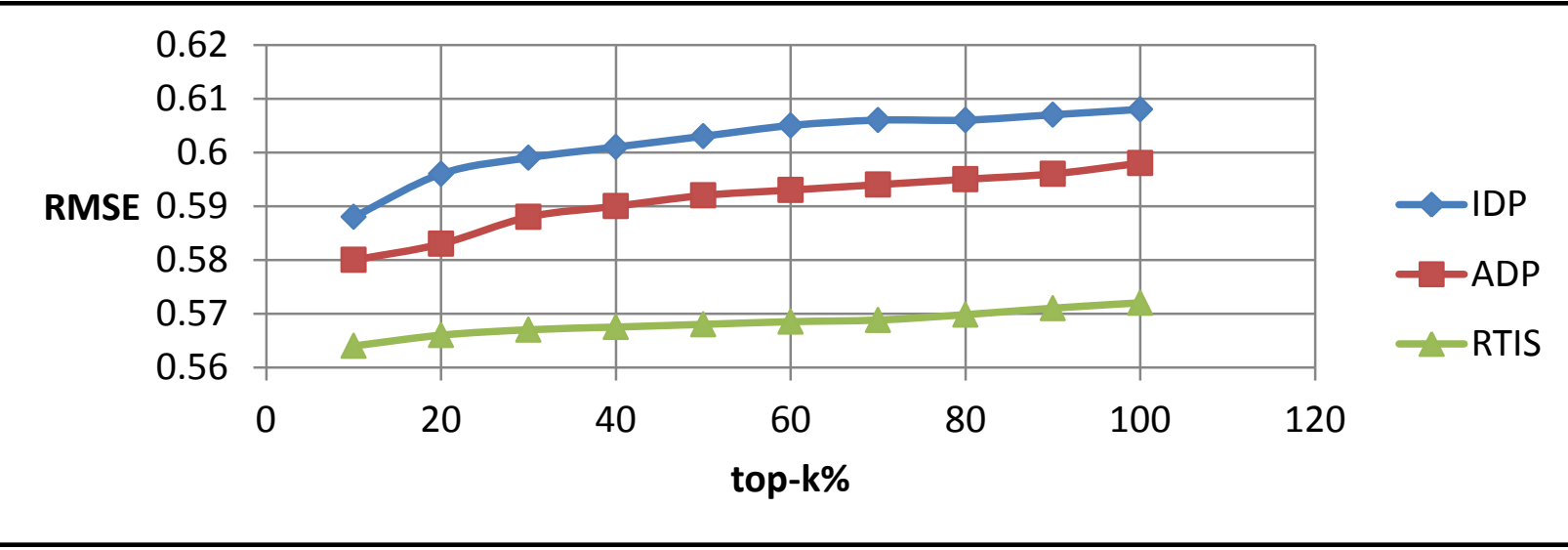

Figure 6 Comparison of different prediction models of proposed model

\section{CONCLUSION AND FUTURE WORK \\ 6.1 Conclusion}

This work was started by studying many research papers on the topic of Music Recommendation Systems. On the basis of literature survey done on various methods of music recommendation, it is accomplished that tags are interesting and provide useful information to enhance RS algorithms. Also, it is concluded that a combination of implicit and explicit feedback in a user preference model provides another paradigm for building an effective recommender systems (RS). Further, from the evaluation of the example provided in proposed work, it is determined that the transformation of play counts using the method in existing system, was not appropriate for skewed data. Hence, to cope up with this problem, normalization step is included before transformation step and a novel method of music recommendation is proposed which uses collaborative tagging method with a combination of both feedbacks to build an effective system which provides more efficient recommendations.

\subsection{Future Work}

The proposed work could be further enhanced in future. First, context information can be used to enhance the recommendation results, in addition to tags and ratings. Second, this idea for mining social media tags can be applied to other multimedia recommendations.

\section{REFERENCES}

[1] Marius Kaminskas and Francesco Ricci. "Contextual music information retrieval and recommendation: State of the art and challenges". Elsevier, 2012.
[2] Yading Song, Simon Dixon, and Marcus Pearce. "A Survey of Music Recommendation Systems and Future Perspectives". 9th International Symposium on CMMR,2012.

[3] Alexandra Uitdenbogerd and van Schyndel Ron. A Review of Factors Affecting Music Recommender. In 3rd International Conference on Music Information Retrieval (2002), 2002

[4] F. Ricci, L. Rokach, B. Shapira, P.B. Kantor (Eds.), Recommender Systems Handbook, Springer, 2011.

[5] http://www.last.fm

[6] Robin Burke. Hybrid Recommender Systems: Survey and Experiments. User Modeling and User-Adapted Interaction, 12(4):331-370, 2002.

[7] Dmitry Bogdanov and Perfecto Herrera. How Much Metadata Do We Need in Music Recommnendation? A Subjective Evaluation Using Preference Sets. In 12th International Society for Music Information Retrieval Conference, number ISMIR 2011, pages 97-102, 2011.

[8] G. Adomavicius, B. Mobasher, F. Ricci, A. Tuzhilin, Contextaware recommender systems, AI Magazine 32 (3) (2011) 67-80.

[9] J.Y. Kim, N.J. Belkin, Categories of music description and search terms and phrases used by non-music experts, in: 3rd International Conference on Music Information Retrieval, Paris, France, 2002, pp. 209-214.

[10] Tso-Sutter K.H. L, Marinho L.B. and Schmidt-Thieme L. 
"Tag-aware recommender systems by fusion of collaborative filtering algorithms". Proc. Int. Conf. on the ACM symposium on Applied computing, Fortaleza, pp. 1995-1999, 2008.

[11] Gunjan Advani and Neha Soni, "A Novel Way for Personalized Music Recommendation Using Social Media Tags”, IJSRD - International Journal for Scientific Research \& Development ,Vol. 2, Issue 11, 2015.

[12] Ja-Hwung Su, Wei-Yi Chang and Vincent S. Tseng. "Personalized Music Recommendation by Mining Social Media Tags". 17th International Conference in Knowledge Based and Intelligent Information and Engineering Systems - KES2013, Elsevier, 2009.

[13] P. Symeonidis, M. Ruxanda, A. Nanopoulos, Y. Manolopoulos, Ternary semantic analysis of social tags for personalizedmusic recommendation, in: Proceedings of 9th International Conference on Music Information Retrieval, Philadelphia, USA, 2008, pp. 219-224.

[14] Qi Q., Chen Z., Liu J., Hui C. and Wu Q. "Using inferred tag ratings to improve user-based collaborative filtering". Proc. of the 27th Annual ACM Symposium on Applied Computin, pp. 2008-2013, 2012.

[15] Jawaheer G., Szomszor M., and Kostkova P. "Comparison of implicit and explicit feedback from an online music recommendation service". ACM Recommender Systems Conference 2010, Barcelona, 2010.

[16] Jawaheer G., Szomszor M., and Kostkova P. "Characterisation of explicit feedback in an online music recommendation service". ACM Recommender Systems Conference 2010, Barcelona, 2010. 